\title{
Binding activity of H-Ras is necessary for in vivo inhibition of ASK1 activity
}

\author{
Jun $\mathrm{DU}^{1,2^{*}}$, Shao Hui $\mathrm{CAI}^{3,4}$, Zhe $\mathrm{SHI}^{3}$, Fumihiko NAGASE ${ }^{1}$ \\ ${ }^{1}$ Department of Medical Technology, Nagoya University School of Health Sciences, Aichi 461-8673, Japan. \\ ${ }^{2}$ School of Pharmaceutical Science, Sun Yat-Sen University, Guangzhou, 510089, China. \\ ${ }^{3}$ Department of Immunology, Nagoya University Graduate and Faculty School of Medicine, Aichi, 466-8550, Japan. \\ ${ }^{4}$ College of Pharmacy, Jinan University, Guangzhou, 510632, China.
}

\begin{abstract}
H-Ras is well known as one of the essential components of Ras/Raf/MEK/ERK cascade, which is a critical prosurvival signaling mechanism in most eukaryotic cells. Ras targets Raf/MEK/ERK cascade by integrating and transmitting extracellular signals from growth factor receptors to Raf, leading to the propagation of signals to modulate a serious of cellular survival events. Apoptosis signal-regulating kinase1 (ASK1) serves as a general mediator of cell death because it is responsive to a variety of death signals. In this study, we found that H-Ras interacted with ASK1 to cause the inhibition of both ASK1 activity and ASK1-induced apoptosis in vivo, which was reversed only partially by addition of RafS621A, an antagonist of Raf, whereas MEK inhibitor, PD98059, and PI3K inhibitor, LY294002, did not disturb the inhibitory effect of H-Ras on ASK-1-induced apoptosis. Furthermore, by means of immunoprecipitate and kinase assays, we demonstrated that the interaction between H-Ras and ASK1 as well as the inhibition of ASK1 activity were dependent on the binding activity of H-Ras. These results suggest that a novel mechanism may be involved in H-Rasmediated cell survival in addition to the well established MEK/ERK and PI3K/Akt kinase-dependent enhancement of cell survival.
\end{abstract}

Keywords: H-Ras, ASK1, apoptosis, binding activity.

\section{INTRODUCTION}

In response to diverse extracellular stimuli such as peptide growth factors, cytokines, hormones and a variety of stresses, cells constantly face dual complete opposite selections: survival or death. Diverse signaling pathways, including those mediated by tyrosine kinase receptors and heterotrimeric G protein-coupled receptors take part in the decision of cell fate[1]. Ras/Raf/MEK/ERK cascade is well known as a critical prosurvival signaling mechanism. HRas, a small guanosine triphosphate-binding protein encoded by H-Ras proto-oncogene, is one of the essential components in this pathway[2]. It is believed that H-Ras is involved centrally in diverse prosurvival signaling transduction pathways and acts as a key regulator of cell growth in all eukaryotic cells[3]. H-Ras targets Raf/MEK/ERK cascade by integrating and transmitting extracellular signals from growth factor receptors to Raf (a MAPKK kinase, MAPKKK). Upon activation, Raf relays to phosphorylate mitogen-activated protein kinase kinase (MEK), which in turn activates mitogen-activated protein kinase/extracellu-

"Correspondence: Jun DU,

Tel: +81(52)719-1547; Fax: +81(52)719-1547;

E-mail: togun@met.nagoya-u.ac.jp lar signal-regulated kinases (MAPK/ERKs), leading to the propagation of signals to modulate a serious of cellular survival events $[4,5]$.

Apoptosis-signal regulating kinase 1 (ASK1) is one of MAP kinase kinase kinases (MAPKKK), which serves as a general mediator of cell death because it is responsive to a variety of death signals[6], including oxidative stresses, treatment of TNF $\alpha$ [7], methylglyoxal[8] (a physiological metabolite), and the chemotherapeutic drugs cisplatin and paclitaxal $[9,10]$. As an upstream activator of the stress responsive protein kinases, ASK1 activates the JNK and p38 MAP kinase cascades and induce apoptosis during exposure to oxidative stress and other stressful stimuli. Regulation of ASK1 by both pro-apoptotic and antiapoptotic signals may provide a critical point of control for cell death and cell survival.

In recent years, the functional "cross-talk" between the prosurvival signaling pathway and pro-apoptosis signaling pathway and the related biological significance to decide cell fate have been understood. For example, it was proved that Raf- 1 could bind to the N-terminal regulatory fragment of ASK1 and decreased the activity of ASK1, which resulted in inhibiting apoptosis mediated by ASK1 signaling pathway and promoting cell survival[11]. Since H-Ras 
acts as a direct upstream regulator of Raf and plays the key role in triggering cellular survival signaling, we were interested in inquiring whether ASK1 was also a potential target of H-Ras. In this report, we have demonstrated that ASK1 really acted as a potential H-Ras target and its activity was suppressed by H-Ras, which leaded to selectively inhibition of ASK1-mediated pro-apoptotic signal pathway, suggesting a novel prosurvival mechanism of action for $\mathrm{H}-$ Ras.

\section{MATERIALS AND METHODS}

\section{Antibodies and chemical reagents}

Anti-MKK3, anti-ERK1, anti-p38, anti-HA, anti-H-Ras antibodies, anti- $\beta$-actin and Protein $A / G$ plus-agarose were purchased from Santa Cruz Biotechnology. Inc.(Santa Cruz, CA). Antiphospho-MKK3 and anti-phospho-ERK1/2 antibodies, PD98059, LY294002, ATF2 peptide and full length JNK1 protein were purchased from New England Biolabs, Inc. (Beverly, MA). GST-MKK4 protein was from Transduction Lab (Lexington, KY). FUGENE ${ }^{\mathrm{TM}} 6$ Transfection Reagent was from Roche Molecular Biochemicals (Mannheim, Germany).

\section{Plasmids}

The constructed pCMV vectors respectively expressing the wildtype H-Ras protein (H-Ras WT), constitutively active form of the $\mathrm{H}$ Ras protein that contains a glycine-to-valine mutation at residue 12 (H-Ras V12) and dominant-negative form of the H-Ras protein that contains a serine-to-asparagine mutation at residue 17 (H-Ras N17), dominant-negative form of the Raf protein that contains a serine-toalanine mutation at residue 621 (Raf S621A), were purchased from Clontech Laboratories Inc. (Palo Alto, CA). pcDNA3-HA-ASK1/ WT and pcDNA3-HA-ASK1/KM plasmids expressing wild-type or dominant negative ASK1 kinases were a generous gift from Dr. H Ichijo (Tokyo Medical and Dental University).

\section{Cell culture, treatment and transfection}

A human embryonic kidney cell line (HEK293) was maintained in Dulbecco's modified Eagle's medium (DMEM) supplemented with $10 \%$ fetal bovine serum (FBS) and antibiotics at $37^{\circ} \mathrm{C}$ in a $\mathrm{CO}_{2}$ incubator. For transfection, cells were plated at a density of $1 \times 10^{4}$ cells $/ \mathrm{cm}^{2}$. After $24 \mathrm{~h}$ of culture, the cells were transfected with plasmid DNA ( $0.1 \mu \mathrm{g}$ of DNA $/ \mathrm{cm}^{2}$ per plasmid) and FUGENE6 reagent according to the instructions of the manufacturer. Assays were performed at $36 \mathrm{~h}$ after transfection. At the end of all treatments, the cells were washed twice with ice-cold PBS and then lysed for whole cell lysate preparations.

\section{Immunoprecipitation and immunoblot analysis}

Cells were lysed in lysis buffer containing 1\% Nonidet P-40, 20 mM Tris- $\mathrm{HCl}$ (pH 7.6), $0.15 \mathrm{M} \mathrm{NaCl}, 3$ mM EDTA, 3 mM EGTA, $1 \mathrm{mM}$ phenylmethylsulfonyl fluoride, $2 \mathrm{mM}$ sodium vanadate, 20 $\mathrm{mg} / \mathrm{ml}$ aprotinin and $5 \mathrm{mg} / \mathrm{ml}$ leupeptin. Insoluble material was removed by centrifugation $\left(12,000 \mathrm{rpm} 30 \mathrm{~min}, 4^{\circ} \mathrm{C}\right)$. Immunoprecipitation was performed by a rat anti-H-Ras monoclonal antibody. The immuno-complexes were collected following incubation with pro- tein A/G plus-agarose beads. For Immunoblotting, the cell lysates or immunoprecipitates were separated on $10 \%$ or $12 \%$ SDS-polyacrylamide gels, and blotted onto a nitrocellulose membrane. Nonspecific binding was blocked by incubating the membrane with 0 . 05\% Tween-20/PBS containing 5\% non-fat dry milk for $1 \mathrm{~h}$ at room temperature. Membranes were incubated with the primary antibody overnight at $4{ }^{\circ} \mathrm{C}$ and then with a horseradish peroxidase-conjugated secondary antibody, and the specific immune complexes were detected using Western Blot Plus Chemiluminescence Reagent (Life Science, Inc, Boston, MA).

\section{Flow cytometric analysis}

For analysis of DNA fragmentation, HEK293 cells were plated onto a 24-well tissue culture plate and transfected with indicated plasmids for $36 \mathrm{~h}$. The cells were starved in a FBS free medium. Twenty-four hours after starvation, total cells were harvested, resuspended in $500 \mu 1$ of propidium iodide buffer $(0.1 \%$ Triton X- 100 , $0.1 \%$ Trisodium citrate) containing $50 \mu \mathrm{g} / \mathrm{ml}$ propidium iodide, and incubated for $15 \mathrm{~min}$ on ice. DNA fragmentation analysis was carried out using a FACScalibur flow cytometer (Becton Dickinson), as described previously[12]. Collected data were processed by the Modfit software, and apoptosis was scored by the percentage of cells appearing in the area below the G1/G0 peak.

\section{ASK1 kinase assay}

The ASK1 kinase assay was performed as described previously [8]. Briefly, the cell extracts were clarified by centrifugation, and the supernatants were immunoprecipitated with HA antibody. The immunocomplexes were bound to protein $\mathrm{A} / \mathrm{G}$ plus-agarose beads. The beads were washed twice with kinase buffer $[20 \mathrm{mM}$ Tris- $\mathrm{HCl}$ ( $\mathrm{pH}$ 7.4), $20 \mathrm{mM} \mathrm{MgCl}_{2}$ ], and subjected to the ASK1 kinase assay. The immunocomplexes were incubated first with $0.1 \mu \mathrm{g}$ of GSTMKK4 for $15 \mathrm{~min}$ at $30^{\circ} \mathrm{C}$ in a final volume of $25 \mu 1$ kinase buffer containing $100 \mu \mathrm{M}$ of ATP, and subsequently with $1 \mu \mathrm{g}$ of JNK1 for $15 \mathrm{~min}$ at $30^{\circ} \mathrm{C}$. Thereafter, the activated complex was incubated with $0.3 \mu \mathrm{Ci}$ of $\left[\gamma^{-32} \mathrm{P}\right] \mathrm{ATP}$ and $1 \mu \mathrm{g}$ of ATF2 peptide in the same solution. The samples were resolved by SDS-PAGE, and the phosphorylation of ATF2 was detected.

\section{RESULTS}

\section{H-Ras inhibited ASk1-induced apoptosis}

To investigate the role of the H-Ras in suppressing ASK1dependent apoptotic signaling, we tested the effect of $\mathrm{H}$ Ras on ASK1-induced apoptosis. HEK293 cells were transiently transfected with human ASK1 alone or together with H-Ras or dominant negative H-Ras N17, in which Ser 17 was replaced by Asn. The cells were stained with PI and DNA fragmentations were analyzed by flowcytometer; and at the same condition, both the expression of ASK1 and H-Ras were detected by immunoblotting with anti-HA and anti-H-Ras antibody respectively. The data showed that the overexpression of ASK 1 alone sufficiently induced cell apoptosis. When cells were co-transfected with $A S K 1$ and H-Ras, however, apoptotic cell death induced by overexpression of ASK1 was largely reduced, 
A

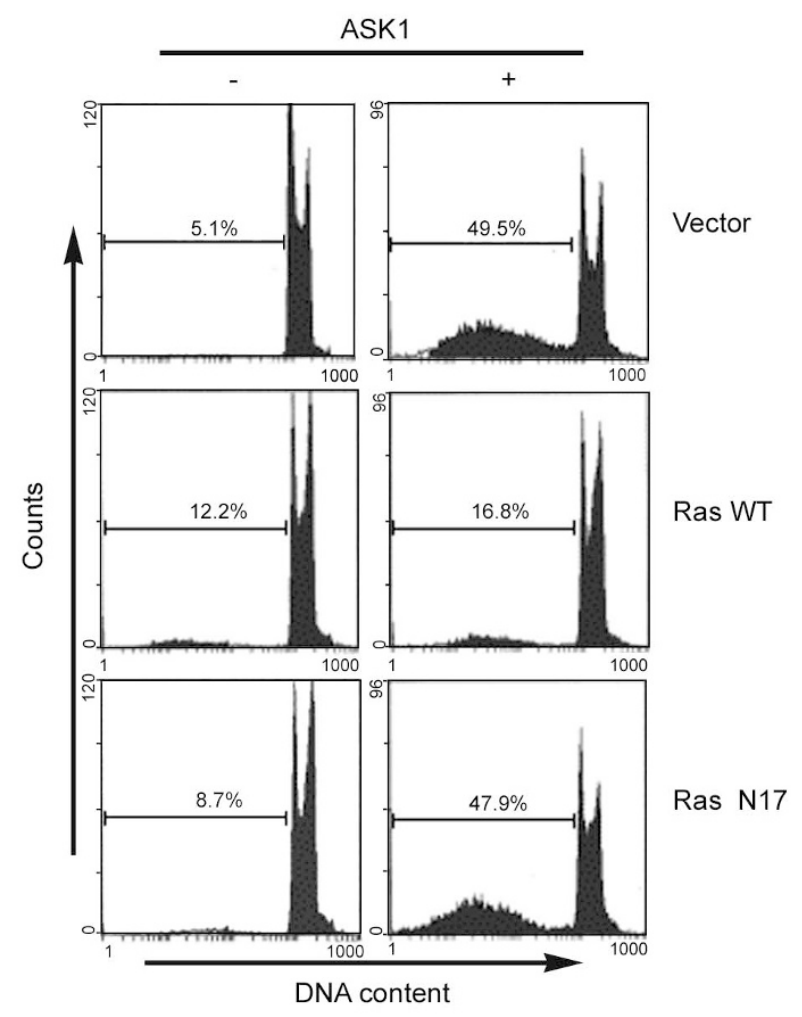

B

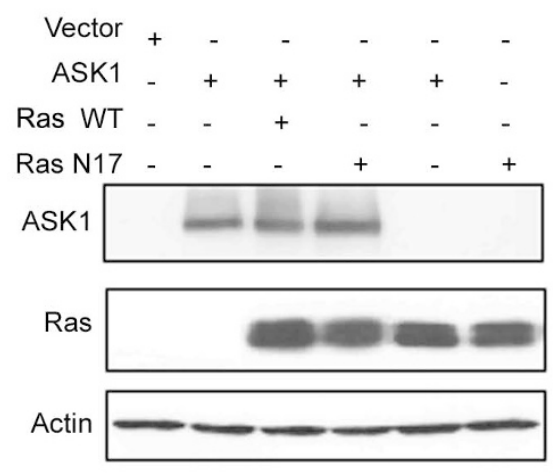

C

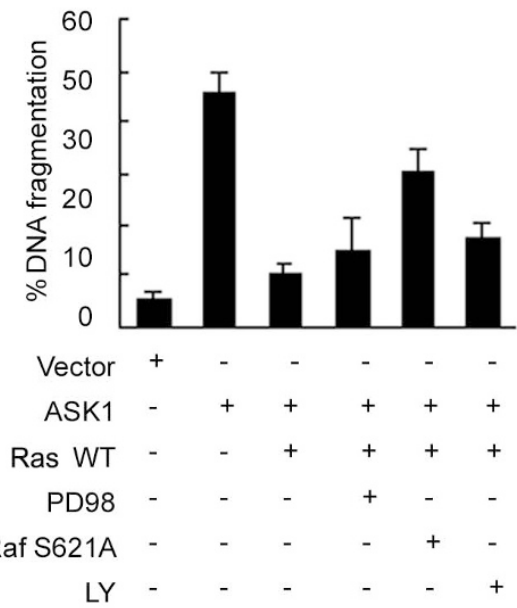

Fig 1. H-Ras suppressed ASK1-induced apoptosis. HEK cells were transfected with or without pcDNA3 vector, pcDNA3/ASK1, pCMV/H-Ras WT or pCMV/H-Ras N17. $36 \mathrm{~h}$ after transfection, the cells were starved in a FBS free medium for $24 \mathrm{~h}$, stained with PI and analyzed by flow cytometer (A). The expression of ASK1 and H-Ras at transfection for $36 \mathrm{~h}$ were detected by immunoblotting with anti-HA and anti-H-Ras antibodies, respectively. $\beta$-actin protein level served as a loading control (B). (C) HEK cells were pretreated with or without $40 \mu \mathrm{M}$ PD98059 (PD98) or $10 \mu \mathrm{M} \mathrm{LY} 294002$ (LY) for $1 \mathrm{~h}$, and then transfected with or without pcDNA3 vector, pcDNA3/ASK1, pCMV/ H-Ras WT or pCMV/H-Raf S621A.

and the DNA fragment approximated to the level of the cells transfected with H-Ras alone (Fig 1A). This inhibitory effect of H-Ras on ASK1-induced cell apoptosis did not appear to be caused by the reduction of ASK1 expression level, because co-transfection with H-Ras did not change the expression amount of ASK1 compared to the cells transfected ASK1 alone, as assessed by immunoblotting assay (Fig 1B). H-Ras-mediated activation of Raf/MEK/ERK and PI3K/Akt cascades were well known as critical prosurvival signaling mechanisms, and some reports indicated that Raf might promote cell survival by antagonizing ASK1 in a Raf-1 protein kinase activity-independent manner[11]. To confirm the hypothesis that whether H-Ras inhibits ASK1-induced apoptosis through Raf/MEK or PI3K/Akt pathway, a negative mutant of Raf (RafS621A), PD98059 (a specific MEK inhibitor) and LY294002 (a specific PI3K inhibitor) were used to block the down-stream effectors of H-Ras in the cells expressing ASK1. As shown in Fig 1C, blocking Raf activity by co-expression of RafS621A, which was believed to weaken the binding ability of Raf, reversed only partially the inhibitory effect of H-Ras. Whereas blocking MEK activity by addition of PD98059 had no obviously change on the inhibitory effect of H-Ras. Moreover, no such inhibition was also observed when cells were treated with LY294002, at a concentration known to abolish PI3K activity. These data suggested that H-Ras inhibited ASK1-induced apoptosis by a MEK/ ERK and PI3K/Akt-independent mechanism, and Raf might partially contribute to the inhibitory process.

\section{H-Ras did not activate the MKK3/p38 kinase pathway}

In the pro-apoptosis signaling cascade, p38 MAPK is 


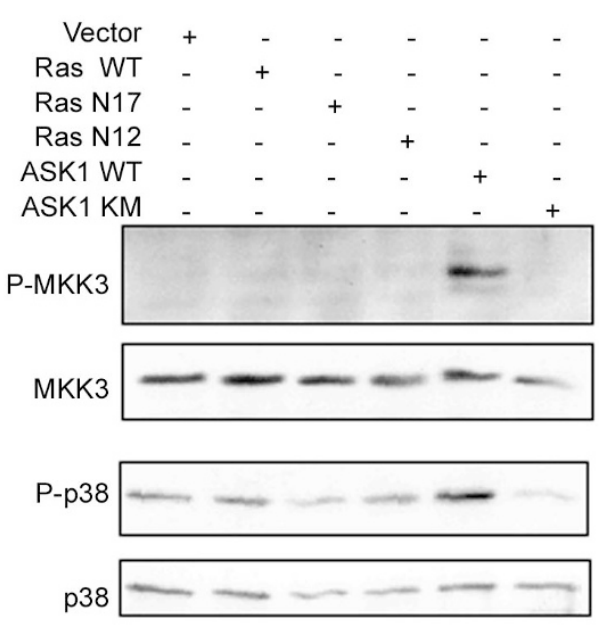

Fig 2. Expression of wild-type ASK1 induced activation of MKK3/ p38 pathway, but not H-Ras. HEK293 cells were transfected with an empty vector or a wild-type H-Ras (Ras WT), dominant negative form of H-Ras (Ras N17), active form of H-Ras (Ras V12), wild-type ASK1 (ASK1 WT), dominant negative form of ASK1 (ASK1 KM) for $38 \mathrm{~h}$ and the activation of MKK3 and p38 were detected by Western blotting with anti-phospho-MKK3 and antiphospho-p38 antibodies, respectively. The membranes were stripped of antibodies and immunoblotted with anti-MKK3 and anti-p38 antibodies (lower panel, respectively).

an important down-stream component of ASK1 in response to diverse extracellular stresses stimuli. Therefore, it is necessary to investigate whether MKK3/p38 pathway is also the target of H-Ras. HEK293 cells were transiently transfected with wild-type (H-Ras WT), dominant negative (H-Ras N17) and active form (H-Ras V12) of H-Ras, wild-type (ASK1 WT) and kinase-inactive form (ASK1 $\mathrm{KM}$, in which Lys 709 was replaced by Met) of ASK1, respectively. The MKK3 and p38 kinase activity was measured by Western blotting with specific antibodies. Unlike wild-type ASK1, which could markedly increase the phosphorylation of both MKK3 and p38 MAPK, the expression of either wild $\mathrm{H}$-Ras or mutated $\mathrm{H}$-Ras failed to stimulate the phosphorylation of MKK3 and p38 MAPK (Fig 2), demonstrating that downstream signaling pathways of $\mathrm{H}$-Ras were insufficient to induce MKK 3 and p 38 activation. In other terms, both MKK3 and p38 were not the direct targets of H-Ras.

\section{H-Ras suppressed ASK1-mediated activation of p38 MAPK}

We next examined whether H-Ras activation is sufficient to affect ASK1-mediated activation of p38 MAPK in vivo. Wild type and mutated H-Ras were co-transfected with ASK1 WT, and phosphorylation of the p38 under serum-free conditions was determined by Western blotting.

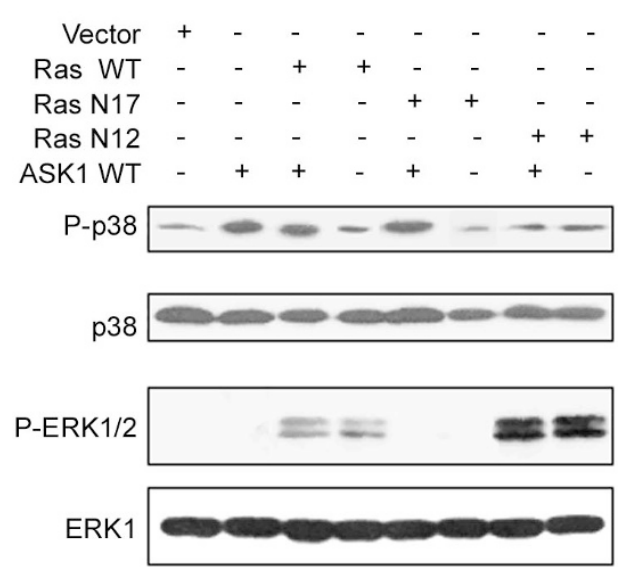

Fig 3. Co-expression of active form H-Ras suppressed wild-type ASK1-induced activation of p38 kinase. HEK293 cells were transiently transfected with the ASK1 WT either together with or without H-Ras wt, H-Ras N17 and H-Ras V12, respectively. The phosphorylated p38 and ERK, total protein level of p38 and ERK were detected by Western blotting with the anti-phospho-p38, -phosphoERK, -p38 and -ERK antibodies.

While H-Ras WT moderately inhibited the phosphorylation of 38 MAPK in response to ASK1 expression, the active form of H-Ras, H-Ras V12, led to clear inhibition of phosphorylation of $\mathrm{p} 38$, whereas co-expression of H-Ras N17 with ASK1 failed to suppress ASK1-mediated p38 phosphorylation (Fig 3). In contrast, co-expression of ASK1 did not alter ERK1/2 phosphorylation state induced by expression of wild-type and active form of H-Ras (Fig 3). Similar results were also observed in the treatment with $\mathrm{H}_{2} \mathrm{O}_{2}$ that has been believed to activate $\mathrm{p} 38$ MAPK through ASK1-mediated signaling. It was found that the active $\mathrm{H}-$ Ras but not binding-defective mutant $\mathrm{H}$-Ras N17 could oppose the effect of $\mathrm{H}_{2} \mathrm{O}_{2}$ on increasing the ASK1-mediated phosphorylation of p38 MAPK (Fig 4). These data imply that H-Ras-inhibited signaling from ASK1 to p38 may be through a target binding mechanism.

\section{Inhibition of ASK1 signaling was relative to the binding activity of $\mathbf{H}$-Ras}

Since binding to its targets is one of the mechanisms for H-Ras to affect a number of downstream signaling states, one possible mechanism for H-Ras to inhibit apoptotic activity of ASK1 is through direct interaction between the two proteins. To test this hypothesis, the plasmids encoding H-Ras WT, H-Ras N17 and H-Ras V12 were respectively co-transfected into HEK293 cells with HAtagged ASK1, and the binding was determined by co-immunoprecipitation from serum-starved cells. As shown in Fig 5, ASK1 was present mainly in H-Ras V12 immunoprecipitate and moderately in H-Ras WT immunoprecipitate 


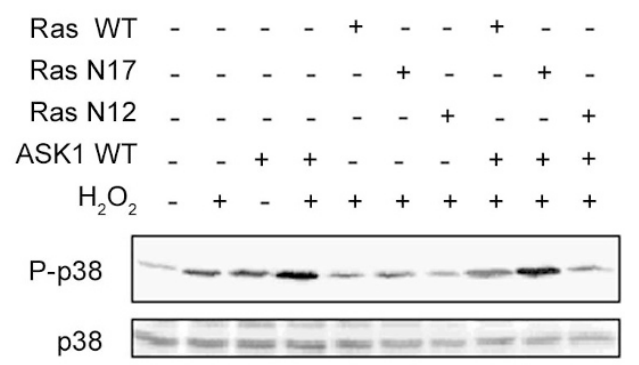

Fig 4. Co-expression of active form of $\mathrm{H}$-Ras suppressed $\mathrm{H}_{2} \mathrm{O}_{2}$ mediated activation of p38 MAPK. HEK293 cells were transiently transfected with the ASK1 WT together with or without H-Ras WT, H-Ras N17 and H-Ras V12 for 36 h, and then treated with 200 $\mathrm{mM}$ of $\mathrm{H}_{2} \mathrm{O}_{2}$ for $30 \mathrm{~min}$. The phosphorylated p38 and the total protein level of $\mathrm{p} 38$ were detected by Western blotting with antiphospho-p38 and anti-p38 antibodies.

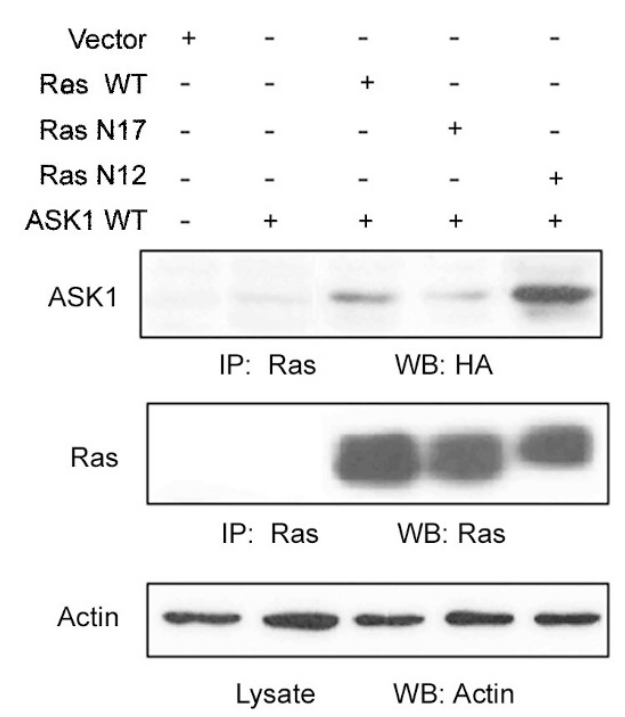

Fig 5. Active form of H-Ras, but not binding activity-defective form of H-Ras, binded to ASK1. HEK293 cells were transiently transfected with ASK1 WT together with or without H-Ras WT, H-Ras $\mathrm{N} 17$ and H-Ras V12 for $36 \mathrm{~h}$. The cells were lysed and subjected to immunoprecipitate with a rat anti-H-Ras antibody. The amount of ASK1 and H-Ras in the immune complex was respectively detected by Western blotting with rabbit anti-HA and mouse anti-H-Ras antibodies. The $\beta$-actin protein level in the lysates was shown (lower panel) as loading control.

but absent in H-Ras N17 immunoprecipitate, indicating that the association between H-Ras and ASK1 was dependent on the binding activity of H-Ras. Furthermore, we examined whether H-Ras and ASK1 interaction was sufficient for inhibiting activity of ASK1. The activity of ASK1 in various immune complexes was measured by a couple kinase assay. The result indicated that co-expression of either H-Ras WT or H-Ras V12 with ASK1 appeared to strongly inhibit the activation of ASK1. In contrast, co-

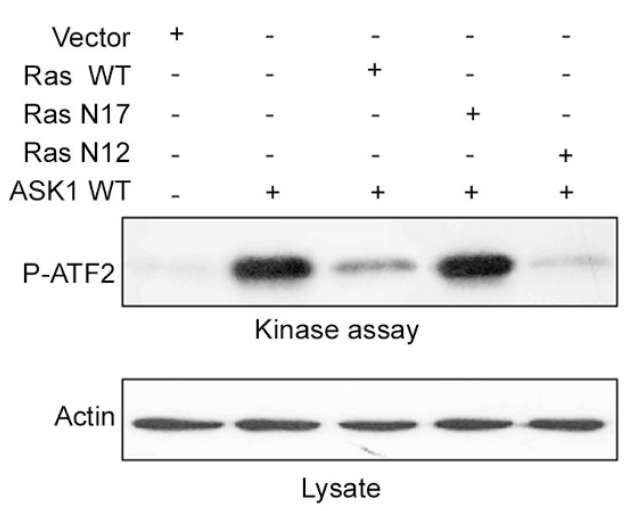

Fig 6. Active form of H-Ras inhibited ASK1 activation. HEK293 cells were transiently transfected with ASK1 WT either together with or without empty vector, H-Ras WT, H-Ras N17 and H-Ras V12 for $36 \mathrm{~h}$. The ASK1 activity was measured by a couple kinase assay as described in Materials and Methods. The $\beta$-actin protein level in the lysates was shown (lower panel).

expression of binding-defective form of H-Ras, H-Ras N17, had no effect on the activity of ASK1 (Fig 6). Taken together, these findings imply that the binding activity of $\mathrm{H}$-Ras is necessary for negatively regulating the function of ASK1.

\section{DISCUSSION}

Recently, growing evidences have shown that the functional "cross-talk" frequently take place between the prosurvival signaling pathway and pro-apoptosis signaling pathway. The relative mechanisms appear to be complex because it is possible that a variety of signaling cascades from these two functional antagonistic signaling pathways get involved in the processes. It has been postulated that apoptotic cell death is the default program of metazoan cells, which must be suppressed continuously by survival mechanisms[13]. In most eukaryotes, $\mathrm{H}$-Ras is a vital component of a variety of growth factor-induced signaling pathways and plays an important role in integrating and transmitting extracellular signals to cellular prosurvival signaling pathways. Generally, H-Ras functions as a positive regulator of the ERK/MAPK signal transduction cascade by activating MEKKs, including Raf-1 and MEKK1. Our findings indicated that there existed another mechanism in which H-Ras promoted cell survival through a downstream kinases activity- independent fashion. We found that ASK1, a critical component of the cellular pro-apoptotic machinery, also acted as a potential target of H-Ras and ASK1-mediated cell death signal was blocked by the interaction of wild-type and active form of $\mathrm{H}$-Ras protein, suggesting a novel prosurvival mechanism for H-Ras. This finding supports the observation that ASK1-mediated cell death is suppressed in response to growth factors, which dominantly 
activate $\operatorname{Ras}[14]$.

It has been reported that Raf-1 could bind to the Nterminal regulatory fragment of ASK 1 and decreased the activity of ASK1, which resulted in inhibiting the apoptosis mediated by ASK1 and promoting cell survival mediated by Raf-1[11]. Therefore, Raf plays the key role not only in triggering cellular survival signaling mediated by Raf/ MEK/ERK cascade but also in inhibiting directly ASK1mediated pro-apoptotic pathway. Since H-Ras acts as a direct upstream activator of Raf, the activation of MEK/ ERK and Raf induced by expression of H-Ras might be responsible for the inhibitory action of H-Ras in ASK1induced apoptosis. In other terms, the regulative effect of H-Ras on ASK1-mediated pro-apoptotic signaling pathway might be dependent on Raf or Raf/MEK/ERK signaling cascade. We provided evidence contradicting this view. Addition of MEK inhibitor, PD98059, at a concentration known to abolish MEK activity, did not block the effect of H-Ras on the inhibition of ASK-1-induced apoptosis. This result indicated that the activation of MEK/ERK, which caused by over-expression of wild-type and active form of H-Ras, is not sufficient for inhibiting ASK-1-induced apoptosis. In addition, inhibition of PI3K, an established downstream target of H-Ras, did not limit the effect of HRas the on the inhibition of ASK-1-induced apoptosis. Our results also showed that the co-expression of a dominant negative form of Raf-1 only weakly reversed the action of H-Ras, suggesting that Raf-1 was involved partially in the inhibition of ASK-1-induced apoptosis. There must be other other signaling pathways, which are closely relative to $\mathrm{H}-$ Ras binding activity, take part in the process.

In this study, we found that ASK1 was presented in wild-type and active form of H-Ras immuno-complexes, but not in that of the binding activity-defective form of $\mathrm{H}$ Ras, H-Ras N17. Moreover, ASK1 activity was reduced in the same manner. These results indicated that physical association of H-Ras might be required for inhibition of ASK1 activity, and subsequent to block ASK1-induced apoptosis.

How the interaction of H-Ras-ASK1 causes the down regulation of ASK1 activity remains unclear. Previous studies indicated that ASK1 directly interacted with its regulators and the ASK1 activity decreased with the separating of ASK1 homo-oligomerization. Homo-oligomerization and association with death receptor associated proteins such as TRAF2 are important process for ASK1 activation[15]. ASK1 interacts physically with the reduced form of thioredoxin, a redox-sensitive protein, and is sequestered in an inactive form[16]. Oxidation of thioredoxin by intracellular ROS results in the activation of ASK1, involving homooligomerization and its association with TRAF2. 14-3-3, a phosphoserine-binding molecule, specifically binds to
ASK1 via Ser-967 of ASK1 and results in inhibition of ASK1-induced apoptosis. Binding to ASK1 by co-expression of a ligand-binding-defective mutant of 14-3-3 causes an increase in homo-oligomerization of ASK1[17]. CDC25, a cell cycle-dependent expression of protein phosphatase, has been reported to bind to C-terminal domain of ASK1 and inhibit its kinase activity. This inhibitory action of CDC25 on ASK1 activity is closely related to diminishing the homo-oligomerization of ASK1[18]. Thus, it seems that the physical association of H-Ras may be inhibitory to this series of events leading to full activation of ASK1. It is possible that $\mathrm{H}$-Ras induces an inactive conformation due to disturbing homo-oligomerization of ASK1. And it is also possible that membrane localization of ASK1 by H-Ras binding will lead to phosphorylation or dephosphorylation of some amino residue(s) on kinases, which might disrupt the interaction of ASK1 with its effectors, causing inactivation of ASK1. Further studies are needed to clarify the exact mechanism of how the activity of ASK1 is inhibited through its physical interaction with H-Ras.

Received, Jul 23, 2003

Revised, Dec 31, 2003

Accepted, Feb 2, 2004

\section{REFFERENCES}

1 Chang L, Karin M. Mammalian MAP kinase signalling cascades. Nature 2001; 410(6824):37-40.

2 Campbell SL, Khosravi-Far R, Rossman KL, Clark GJ, Der CJ. Increasing complexity of Ras signaling. Oncogene 1998; 17(11): 1395-413.

3 Bonni A, Brunet A, West AE, et al. Cell survival promoted by the Ras-MAPK signaling pathway by transcription-dependent and -independent mechanisms. Science 286(5443): 1358-62.

4 Widmann C, Gibson S, Jarpe MB, Johnson GL. Mitogen-activated protein kinase: conservation of a three-kinase module from yeast to human. Physiol Rev 1999; 79(1):143-80.

5 English J, Pearson G, Wilsbacher J, et al. New insights into the control of MAP kinase pathways. Exp Cell Res 1999; 253(1): 255-70.

6 Ichijo H, Nishida E, Irie K, et al. Induction of apoptosis by ASK1, a mammalian MAPKKK that activates SAPK/JNK and p38 signaling pathways. Science 1997; 275(5296):90-4.

7 Nishitoh H, Saitoh M, Mochida Y, et al. ASK1 is essential for JNK/SAPK activation by TRAF2. Mol Cell 1998; 2(3):389-95.

8 Du J, Suzuki H, Nagase F, et al. Superoxide-mediated early oxidation and activation of ASK1 are important for initiating methylglyoxal-induced apoptosis process. Free Radic Biol Med 2001; 31(4):469-78.

9 Chen Z, Seimiya H, Naito M, et al. ASK1 mediates apoptotic cell death induced by genotoxic stress. Oncogene 1999; 18(1): 173-80.

10 Wang TH, Wang HS, Ichijo H, et al. Microtubule-interfering agents activate c-Jun N-terminal kinase/stress-activated protein kinase through both Ras and apoptosis signal-regulating kinase pathways. J Biol Chem 1998; 273(9):4928-36. 
11 Chen J, Fujii K, Zhang L, Roberts T, Fu H. Raf-1 promotes cell survival by antagonizing apoptosis signal-regulating kinase 1 through a MEK-ERK independent mechanism. Proc Natl Acad Sci USA 2001; 98(14):7783-8.

$12 \mathrm{Du}$ J, Cai S, Suzuki H, et al. Involvement of MEKK1/ERK/ P21Waf1/Cip1 signal transduction pathway in inhibition of IGFI-mediated cell growth response by methylglyoxal. J Cell Biochem 2003; 88(6): 1235-46.

13 Santen RJ, Song RX, McPherson R, et al. The role of mitogenactivated protein (MAP) kinase in breast cancer. J Steroid Biochem Mol Biol 2002; 80(2):239-56.

14 Galvan V, Logvinova A, Sperandio S, et al. IGF-IR signaling inhibits apoptosis signal-regulating kinase 1 (ASK1). J Biol Chem 2003; 278(15):13325-32.
15 Liu H, Nishitoh H, Ichijo H, Kyriakis JM. Activation of apoptosis signal-regulating kinase 1 (ASK1) by tumor necrosis factor receptor associated factor 2 requires prior dissociation of the ASK1 inhibitor thioredoxin. Mol Cell Biol 2000; 20(6):2198-208.

16 Saitoh, M. Nishitoh H, Fujii M, et al. Mammalian thioredoxin is a direct inhibitor of apoptosis signal-regulating kinase (ASK) 1. EMBO J 1998; 17(9):2596-606.

17 Liu Y, Yin G, Surapisitchat J, Berk BC, Min W. Laminar flow inhibits TNF-induced ASK1 activation by preventing dissociation of ASK1 from its inhibitor 14-3-3. J Clin Invest 2001; 107 (7):917-23.

18 Zou X, Tsutsui T, Ray D, et al. The cell cycle-regulatory CDC25A phosphatase inhibits apoptosis signal-regulating kinase 1 . Mol Cell Biol 2001; 21(14):4818-28. 